\title{
A Rare Case of Apophysomyces Variabilis Skin and Soft Tissue Infection
}
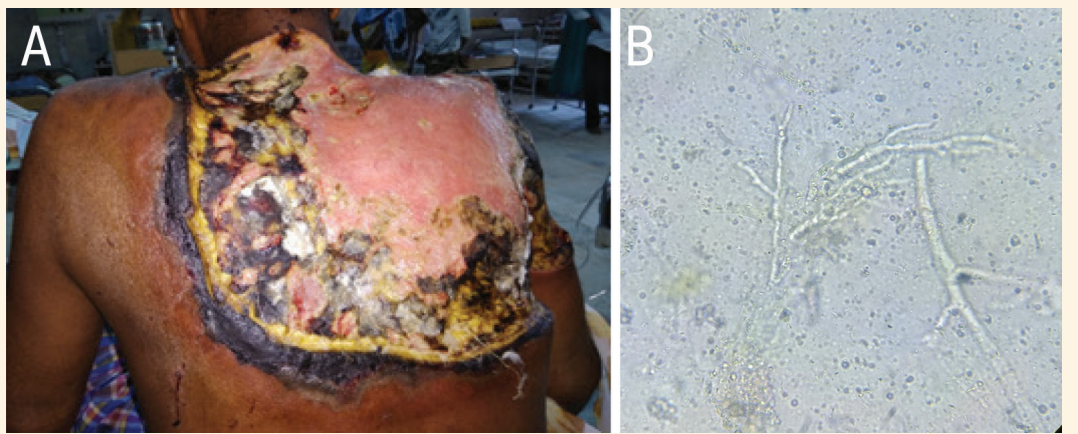

Figure 1: A: Extensive necrotising wound on the back of a 60-year-old male patient who presented to the Department of Surgery, Dr Sampurnanand Medical College in Jodhpur, Rajasthan, India, in 2018, with complaints of pain on his back for one month. B: Potassium hydroxide mount from the biopsy tissue showing broad aseptate hyphae with wide-angle branching.

A 6O-YEAR-OLD MALE PATIENT PRESENTED TO the Department of Surgery, Dr Sampurnanand Medical College in Jodhpur, Rajasthan, India, in 2018, with complaints of back pain for one month (day 0 ). On examination, a boil of size 3 $\times 3 \mathrm{~cm}$ was observed on his back, at the medial side of the left scapular region. He was prescribed amoxicillinclavulanic acid at $625 \mathrm{mg}$ three times a day and nonsteroidal anti-inflammatory drugs for one week. No microbiological evaluation was requested by the treating surgeon on day 0 . The patient presented again on day 5 with complaints of no improvement, and was subsequently hospitalised for three days. Incision and drainage of the wound was done and dressing with antibiotic coverage was given. On day 13, the patient came back again with complaints of no improvement. Wound debridement and dressing was done and the patient was called for follow-up after seven days. The patient once again came in on day 18 with a massive necrotising wound on the back. Debridement of the dead tissue and toileting of the wound was done with antibiotic coverage. The patient was called for another follow-up after seven days. On day 28, the patient presented to the emergency department, febrile and well-oriented to the time, place and person, with an extensive necrotising wound on his back, extending up to the right arm, with a creeping wound margin in his subcutaneous tissue [Figure 1A].
The patient had no history of diabetes mellitus, hypertension or any other chronic illness. Complete blood counts and blood glucose were within normal range. On examination of the wound, a clinical diagnosis of necrotising fasciitis was made. Differential diagnosis for aetiological agents of mucormycosis, subcutaneous mycosis and entomophthoromycosis were made by the attending surgeon. Necrotic tissue was removed, and a skin biopsy sample was sent for bacterial culture and sensitivity, potassium hydroxide $(\mathrm{KOH})$ examination and fungus culture to the microbiology laboratory. Upon $10 \% \mathrm{KOH}$ mount examination, hyaline, broad, aseptate fungal hyphae with wide-angle branching were seen [Figure 1B].

A histopathology examination was not performed and based on $\mathrm{KOH}$ examination findings, the patient was hospitalised. Treatment of intravenous amphotericin B, $5 \mathrm{mg} / \mathrm{kg} /$ day for four weeks, with $5 \%$ dextrose was started immediately along with alternate day amphotericin-B-soaked dressing of the wound. Along with the antifungal agent, antibiotic coverage of piperacillin-tazobactam $4.5 \mathrm{~g}$ every eight hours was also administered for one week.

Bacterial culture was sterile but white cottony fungal growth with aerial mycelia was seen on Sabouraud dextrose agar (SDA) after 48 hours of incubation. A lactophenol cotton blue mount was prepared from the growth on day 33 (five-day- 

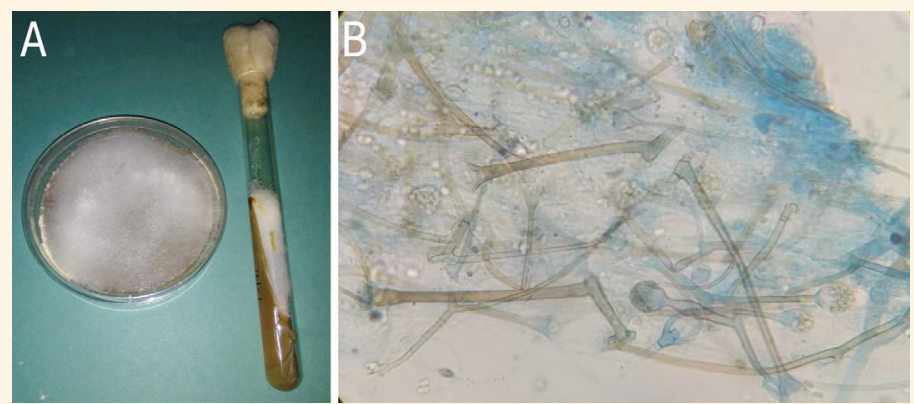

Figure 2: A: Fungal colony on Sabouraud dextrose agar showing aerial hyphae filling the culture plate and tube. B: Lactophenol cotton blue mount of the 15-day-old colony showing broad aseptate hyphae with funnel-shaped sporangiospores arising from the foot cell.

old colony), day 38 (10-day-old colony) and day 43 (15-day-old colony). In the five-day-old colony, only broad aseptate fungal hyphae were seen; in the 10-dayold colony, scanty sporangia were observed; and in the 15-day-old colony, broad aseptate hyphae bearing sporangium with typical funnel-shaped apophysis was observed at 400x magnification [Figure 2].

Sporangiophores were seen to arise from the foot cell. A dark, thick brown structure was observed just below the sporangiophores. The sporangiospores were oblong and 6-8 $\mu \mathrm{m}$ in size. All these features were consistent with Apophysomyces species complex. The culture report was conveyed to the treating surgeon on day 44. Antifungal sensitivity testing was not performed for this isolate.

When fresh granulation tissue started to grow at the wound margin, skin grafting was performed by the surgeon. A skin auto-graft was done on day 57 . The patient was discharged after three days of skin-grafting and was kept on follow-up for one month. The skin graft healed well and there was no sign of recurrence of fungal infection or secondary bacterial infection.

The fungal isolate was identified as Apophysomyces variabilis by internal transcribed spacer region sequencing at the National Culture Collection for Pathogenic Fungi, Chandigarh, India. The GenBank accession number of the nucleotide sequence is MN317265.

The patient provided written informed consent before undergoing surgical procedures and provided consent for his case to be reported for scientific purposes.

\section{Comment}

Although Apophysomyces spp. is an environmental fungus, human infection cases are being increasingly reported worldwide. Apophysomyces have been isolated from the soil of tropical and subtropical regions. While most of the clinical cases have been reported in India, this fungus has also been reported in
Australia, the United States, southeast Asia and South America. ${ }^{1,2}$ A. variabilis was first described in 2010, based on the analysis of the sequences of the histone 3 gene, the internal transcribed spacer region of the rDNA gene, and domains D1 and D2 of the 28S rRNA gene. Based on these genetic studies, Apophysomyces species complex has been differentiated into A. elegans, A. variabilis, A. ossiformis, and A. trapeziformis. Phenotypically, differences in apophysis and sporangiospores are observed among these species. A. elegans shows bell and funnel-shaped apophyses and ovoid, subspherical, broadly ellipsoidal to barrel-shaped sporangiospores; A. variabilis shows funnel-shaped apophysis and clavate to ellipsoidal sporangiospores; A. ossiformis shows funnel-shaped apophysis and biconcave sporangiospores; and A. trapeziformis shows funnelshaped apophysis and smaller, trapezoid-shaped sporangiospores when viewed from the side. ${ }^{2}$

Although cases of sporulation of A. spp. in primary cultures on SDA have not been reported in the literature, in one study, sporulation was observed after two days of incubation on primary culture media such as SDA and brain heart infusion agar. ${ }^{1}$ The isolate in this study showed noticeable sporulation on SDA in primary culture only after two weeks of incubation. Subsequent subcultures on SDA also showed abundant sporulation after two weeks of incubation.

Necrotising fasciitis is a severe soft tissue infections caused by bacterial as well as fungal pathogens. The necrotising infections are found mainly in immunocompetent patients. Some fungi of the order Mucorales have an invasive property and can produce this rapidly progressive infection, which destroys the soft tissues and spreads along the fascial planes and invades deeper into the tissues. ${ }^{3}$ While most common fungal aetiology of necrotising fasciitis is linked to Rhizopus spp., other Mucoralean species that are associated with human infection include Mucor, Rhizomucor, Lichtheimia, Apophysomyces, Saksenaea, Cunninghamella, Cokeromyces and Syncephalastrum spp. ${ }^{4}$ These fungal infections are often fatal, even if 
treated in a timely manner. ${ }^{3}$ The introduction of soil contaminated with such organisms may serve as the primary means of inoculation of this organism at the trauma site. Iatrogenic soft tissue mucormycosis after skin graft, renal transplant, orthopaedic surgery and lower segment caesarean section have been reported in the literature. ${ }^{5}$

The current case highlights that $A$. variabilis infection may present as boils but possible inoculation of fungal agent iatrogenically cannot be ruled out in this case. Although the case in early stage was refractory to broad spectrum antibiotics, culture and sensitivity at this stage would have helped to conclude the exact aetiology and progression of the case of the boil to necrotising fasciitis.

\section{AUTHORS' CONTRIBUTION}

$\mathrm{AB}, \mathrm{LR}$ and GSC were involved in data collection. VKM analysed the data. AB and LR contributed with the laboratory investigations. LR and VKM were involved in the literature review. GSC contributed to the clinical study. PKK provided resources and supervised the work. VKM and GSC drafted the initial manuscript. $\mathrm{AB}$ and PKK edited the manuscript. All authors approved the final version of the manuscript.

\section{References}

1. Chander J, Stchigel AM, Alastruey-Izquierdo A, Jayant M, Bala K, Rani $\mathrm{H}$, et al. Fungal necrotizing fasciitis, an emerging infectious disease caused by Apophysomyces (Mucorales). Rev Iberoam Micol 2015; 32:93-8. https://doi.org/10.1016/j.riam.2014.06.005.

2. Alvarez E, Stchigel AM, Cano J, Sutton DA, Fothergill AW, Chander J, et al. Molecular phylogenetic diversity of the emerging mucoralean fungus Apophysomyces: Proposal of three new species. Rev Iberoam Micol 2010; 27:80-9. https://doi.org/10.1 016/j.riam.2010.01.006

3. Skiada A, Petrikkos G. Cutaneous zygomycosis. Clin Microbiol Infect 2009; 15:41-5. https://doi.org/10.1111/j.1469-0691.2009.02979.x.

4. Jain D, Kumar Y, Vasishta RK, Rajesh L, Pattari SK, Chakrabarti A. Zygomycotic necrotizing fasciitis in immunocompetent patients: a series of 18 cases. Mod Pathol 2006; 19:1221-6. https://doi. org/10.1038/modpathol.3800639.

5. Mathews MS, Raman A, Nair A. Nosocomial zygomycotic postsurgical necrotizing fasciitis in a healthy adult caused by Apophysomyces elegans in South India. Med Mycol 1997; 35:61-3. https://doi.org/10.1080/02681219780000891. 